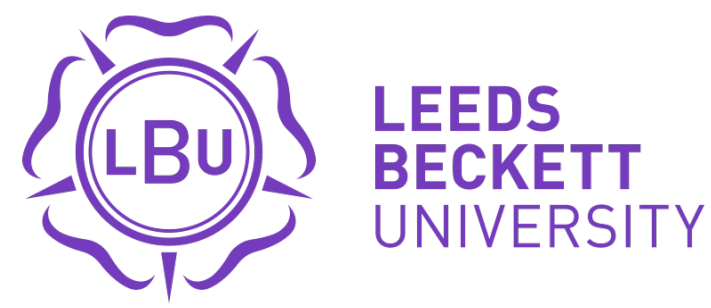

Citation:

Traviss-Turner, GD and Philpot, $U$ and Wilton, $J$ and Green, $K$ and Heywood-Everett, $S$ and Hill, AJ (2018) Guided self-help to manage binge eating in a dietetic-led community weight management service. Clinical Obesity, 8 (4). pp. 250-257. ISSN 1758-8111 DOI: https://doi.org/10.1111/cob.12259

Link to Leeds Beckett Repository record:

https://eprints.leedsbeckett.ac.uk/id/eprint/8266/

Document Version:

Article (Accepted Version)

This is the peer reviewed version of the following article: Traviss-Turner, G.D., Philpot, U., Wilton, J., Green, K., Heywood-Everett, S. and Hill, A.J. (2018), Guided self-help to manage binge eating in a dietetic-led community weight management service. Clin Obes, 8: 250-257, which has been published in final form at https://onlinelibrary.wiley.com/doi/10.1111/cob.12259. This article may be used for non-commercial purposes in accordance with Wiley Terms and Conditions for Use of Self-Archived Versions.

The aim of the Leeds Beckett Repository is to provide open access to our research, as required by funder policies and permitted by publishers and copyright law.

The Leeds Beckett repository holds a wide range of publications, each of which has been checked for copyright and the relevant embargo period has been applied by the Research Services team.

We operate on a standard take-down policy. If you are the author or publisher of an output and you would like it removed from the repository, please contact us and we will investigate on a case-by-case basis.

Each thesis in the repository has been cleared where necessary by the author for third party copyright. If you would like a thesis to be removed from the repository or believe there is an issue with copyright, please contact us on openaccess@leedsbeckett.ac.uk and we will investigate on a case-by-case basis. 
Guided self-help to manage binge eating in a dietetic-led community weight management service

Traviss-Turner, Gemma, D., ${ }^{\mathrm{a}}$ Philpot, Ursula, ${ }^{\mathrm{b}}$ Wilton, Janine, ${ }^{\mathrm{c}}$ Green, Kathleen, ${ }^{\mathrm{b}}$ HeywoodEverett, Suzanne, ${ }^{\text {a Hill, Andrew J. }}{ }^{\text {a }}$

a. Leeds Institute of Health Sciences, School of Medicine, Level 10 Worsley Building, Clarendon Way, University of Leeds, UK

b. School of Clinical \& Applied Sciences, Leeds Beckett University, UK

c. Dietetic Department, Leeds General Infirmary, UK

Corresponding author: Gemma Traviss-Turner G.Traviss@leeds.ac.uk 01133430825

Running title: GSH for binge eating in community dietetics

Key words: Binge eating, Obesity, Weight management, Dietitian, Guided self-help, Wellbeing 


\title{
Guided self-help to manage binge eating in a dietetic-led community weight management service
}

\section{What is already known about this subject?}

- Symptoms of binge eating are common in patients with obesity and a barrier to effective weight management.

- Guided self-help (GSH) is a recommended first stage intervention for binge eating disorder.

- GSH has been successfully delivered by a range of health professionals and dieticians are well placed to be trained as Guides in delivering GSH.

\section{What this study adds?}

- Evidence that a GSH intervention for binge eating can be effective in a community weight management service.

- Dietitians, if trained and supervised in GSH, can lead as Guides in a GSH intervention for binge eating.

- Within treatment assessments are valuable in showing good therapeutic relationships between patients and Guides and that mid-treatment wellbeing was associated with end of treatment outcomes.

\begin{abstract}
An estimated $30 \%$ of patients accessing community weight management services experience symptoms of binge eating disorder (BED). Guided self-help (GSH) is the recommended first line of treatment for BED. This study is a preliminary investigation into the effectiveness of GSH delivered by dietitians for patients with binge eating within a weight management service and a consideration of the association between wellbeing, therapeutic relationship and outcomes. The study was conducted as a single group, pre- and post-intervention study with 24 patients reporting symptoms of binge eating who completed the self-help manual with guidance from a trained community dietitian. Primary outcomes were eating disorder psychopathology and behaviours (EDE-Q), depression and anxiety. Principle results showed a significant reduction on all subscales of eating disorder psychopathology, anxiety and depression. There was a reduction in loss of control over eating but the $40 \%$ reduction in binge episodes was not statistically significant. Mid-treatment sessional ratings were positively associated with outcome. In conclusion, the GSH intervention was appropriate for
\end{abstract}


dietitian delivery to patients with obesity and binge eating behaviour. This research indicates potential for other dietetic-led weight management services to deliver such interventions and support patients with binge eating accessing their service. 


\section{Introduction}

Individuals with binge eating disorder (BED) binge on large amounts of food in a discrete period with accompanying loss of control [1]. BED causes marked distress in individuals, with frequency of binges averaging a minimum of once a week over a three-month period [1]. The lifetime prevalence of BED in the community is approximately $4 \%$ [2], with an estimated prevalence of $30 \%$ in people accessing weight management services [3]. Overweight and obesity, together with the associated physical and psychological health concerns, are commonly comorbid with binge eating [4]. Importantly, individuals with binge eating behaviours do less well in behavioural interventions for weight management $[5,6]$. Binge eating is therefore a barrier to effective weight management in people with obesity. BED has only recently gained full recognition as an eating disorder within DSM-5. Accordingly, recognition of symptoms and the potential for diagnosis may be much lower than that of other eating disorders. Weight management services are likely to be accessed by individuals with BED as they typically, "are heavier, are more likely to be overweight as a child and demonstrate weight cycling" [7]. However, treatments for BED do not result in weight loss, even though they do reduce or remit binge eating [8]. This emphasises the need to address binge eating in settings that have the primary objective of enabling weight loss.

The recently revised NICE guidelines recommend offering a binge eating focused guided self-help (GSH) programme to adults with BED [9]. GSH is a brief, patient-led intervention, which comprises a self-help resource with guidance from a health care professional or lay person. There is a growing body of evidence for the use of GSH as an effective first line intervention for reducing eating disorder psychopathology delivered in mental health settings [10-12]. However, little is known about its delivery in other settings where binge eating presents e.g. weight management services.

Research into the effects of GSH for eating disorders has shown that the Guide plays a significant role $[11,12]$. Intervention outcomes are moderated by the provision of guidance by a health professional, the quality of the guidance, the number of sessions, and eating disorder psychopathology at baseline [11]. The contribution of the Guide appears to depend less on their qualification or profession, and more on their qualities and skills. The role of the Guide has been described as a 'facilitator not therapist', providing emotional support at times of distress and also in 'just being there as a sounding board' [13]. Further information on 
patient/Guide perceptions of the therapeutic relationship in different clinical settings would be extremely valuable.

Dietitians working in weight management services often have skills in advanced behaviour change and communication skills, in addition to individual tailoring of nutrition interventions and helping patients to re-establish a healthy relationship with food [14]. Dietitians working in weight management services therefore are well placed to deliver GSH interventions to reduce binge eating in patients with obesity.

The study aim therefore was to determine the preliminary effectiveness of GSH for patients with binge eating delivered by dietitians within a weight management service. The study trained dietitians in the Working to Overcome Eating Difficulties intervention, an evidencebased GSH approach [15]. A secondary aim was to determine the association between sessional ratings of wellbeing and the perceived therapeutic relationship between patient and dietitian with the main clinical outcomes.

\section{Materials and Methods}

Participants and procedure

This study was a single group, pre-post intervention service evaluation. Patients were recruited from Leeds Community Health Care Trust Adult Community Dietetics Weight Management Service between February 2013 and October 2015. Criteria for referral to the service included: being over 16 years of age, registered with a General Practice in Leeds, having a body mass index (BMI) of over $30 \mathrm{~kg} / \mathrm{m}^{2}$ or a BMI of over $27 \mathrm{~kg} / \mathrm{m}^{2}$ with comorbidities. Patients had a clinical assessment with a dietitian on referral to the service, in which patients were weighed and measured to ascertain BMI using a height measure (SECA 412) and calibrated weighing scales (SECA 761). Binge eating was routinely assessed by dietitians using the DSM criteria (eating large amounts of food in a discrete period and feelings of loss of control over eating). Those reporting at least one episode per week over a 3 month period, were invited to take part in the intervention. One-to-one follow-up sessions with the dietitian or group behavioural weight loss were available to patients after completion of GSH if patients wanted to continue to work on any areas of treatment post intervention, for example portioning food, managing social eating, or fitting in food around shift patterns. Patients were excluded if they were not literate in the English language, lacked capacity to 
read or understand the manual, were at high risk of self-harm or suicide, or were currently abusing drugs or alcohol. A sample size calculation was not conducted because this was a pragmatic service evaluation, conducted over a defined period of time. In total, 50 patients consented to take part in the study, of whom 24 completed the intervention and primary outcome assessments and were included in the main analysis.

This study was conducted according to the guidelines laid down in the Declaration of Helsinki and all procedures involving human patients were approved by Leeds Community Healthcare NHS Trust and Leeds Beckett University, adhering to Leeds Beckett University Research Ethics Policy and Procedures (Ref: 18248, approved on 7/12/15). Written informed consent was obtained from all patients. The reporting of this work is compliant with TREND guidelines for nonrandomized evaluations of behavioral and public health interventions [15].

\section{Intervention}

Each patient who consented to receive GSH attended an introductory session in which they were given a copy of the GSH manual - Working to Overcome Eating Difficulties (for further details on the intervention see [13]). The approach was patient-led and the manual content was based on the principles of cognitive behavioural therapy. The manual comprised six main sections: 1) What are eating disorders, 2) Physical and psychological health, 3) Food, health and unwanted behaviours, 4) Negative thoughts: identifying and challenging, 5) Learning to feel good about you, and 6) Relapse prevention: preparing for the future. Patients were required to complete exercises in the relevant sections prior to each appointment. Their guide (the trained dietitian) would then review their responses and troubleshoot any difficulties they had completing the section. In total, patients received seven, one hour sessions with their guide, spread over 12 weeks. The first four sessions were weekly, followed by two fortnightly, and the final session a month later. This is comparable to delivery of GSH in other settings.

Guides were specifically trained in delivering the intervention, receiving two days group training from two of the authors (UP and SHE). The training develops skills underpinning cognitive behavioural therapy (CBT), and those in psychological formulation, motivational interviewing, setting up behavioural experiments, and working with impasse. Ongoing group supervision was offered throughout the recruitment period at 8 weekly intervals. 
Measures

Outcome measures were collected at baseline and the end of treatment (12 weeks) as part of routine care.

\section{Primary outcome}

Eating disorder psychopathology and key behaviours - (Eating Disorder Evaluation Questionnaire EDE-Q) [17].

This 28 item self-report questionnaire assesses the severity and frequency of symptoms over the past 28 days. It comprises four subscales: restraint, eating concern, shape concern, and weight concern. A global score was calculated by taking the mean of the four subscales. Key behavioural features included objective binge episodes (OBE), vomiting, laxative abuse and excessive exercise. All subscales have shown high internal consistency (Cronbach a coefficients $>0.70)$ and good test-retest reliability $[18,19]$.

\section{Secondary outcomes}

Depression - Patient Health Questionnaire (PHQ-9) [20].

The PHQ-9 is a self-administered 9-item depression-specific questionnaire reflecting on the past 2 weeks. Items are scored from 0 to 3, with higher scores indicating more severe depression.

Anxiety - Generalised Anxiety Disorder Questionnaire (GAD-7) [21].

This is a 7-item clinical self-report measure of generalized anxiety disorder reflecting on symptoms experienced over the past 2 weeks. The measure has good reliability, criterion, construct and factorial validity.

Body Mass Index - was measured and calculated by dietitians $\left(\mathrm{kg} / \mathrm{m}^{2}\right)$.

Sessional assessments:

Sessional assessments were completed at the start and end of every session. The outcome rating scale (ORS) [22] was completed at the start of each session. It assesses the previous week (including 'today'), rating how the patient feels about personal well-being, close interpersonal relationships, social relationships, and overall well-being, in the form of a 
visual analogue rating scale (VAS). Scores range from 1-100mm. The session rating scale (SRS) [22], completed at the end of the session, measures the patient's experience of the session in terms of feeling understood (relationship) agreement on goals and topics, how they found the guide's approach/methods and an overall evaluation of the session, in the same format as the ORS. Both measures have previously demonstrated good reliability and concurrent validity with their longer alternatives [23].

\section{Data analysis}

Data were analysed using the Statistical Software Package for the Social Sciences (SPSS version 22). Significance was assumed when $p<0.05$. The main analyses are presented on a completer basis, however an intention to treat analysis using last observation carried forwards (LOCF) was also conducted. Baseline variables were compared between completers and noncompleters using independent sample t-tests.

Means and standard deviations were calculated for each subscale of the EDE-Q and for frequency of key behaviours at both baseline and end of treatment. Total scores were calculated for secondary outcome measures. Parametric paired-samples t-tests were used to compare baseline and end of treatment scores. Where a distribution was not normally distributed, non-parametric Wilcoxon signed-rank tests were carried out and results compared to t-test output. Bivariate Pearson correlations were used to explore the relationship between sessional ratings at sessions 2, 4 and 6 and outcome (change score on Global EDE-Q). These sessions represent early, mid and late stages of treatment, in line with earlier work [13].

\section{Results}

In total 50 participants consented to take part in the study, of whom 24 (48\%) completed the full intervention and primary outcome assessments and were therefore included in the main analysis. Figure 1 shows the flow of participants through each stage of the study.

Data collected at baseline showed that 44 participants were female (88\%). All were 18 years of age or over. BMI ranged from $30.1 \mathrm{~kg} / \mathrm{m}^{2}$ to $55.4 \mathrm{~kg} / \mathrm{m}^{2}$ (Mean=43.0 $\left.\mathrm{SD}=5.7\right)$. 
There were no differences between completers and non-completers at baseline on BMI, EDEQ restraint, weight concern, shape concern or PHQ-9 total scores. Those who did not complete the intervention scored marginally higher in assessed anxiety $(\mathrm{t}(48)=2.08 ; \mathrm{p}=0.04)$, eating concern $(\mathrm{t}(48)=-2.65 ; \mathrm{p}=0.01)$ and binge frequency $(\mathrm{t}(48)=-2.50 ; \mathrm{p}=0.02)$.

Figure 1 near here

\section{Eating disorder psychopathology, BMI and mood}

Participants completing the intervention reported significant reductions on all the EDE-Q subscales of eating disorder psychopathology (Table 1), along with reductions in loss of control over eating. Binge episodes reduced in frequency by $40 \%$ but the change was not statistically significant. Likewise, the small reduction in BMI did not reach statistical significance. There were significant reductions in depression and anxiety over the course of the intervention.

Intention to treat analysis showed that improvements in all EDE-Q subscales and thus global eating psychopathology $(\mathrm{t}(49)=4.69 ; \mathrm{p}=<0.001)$, loss of control over eating $(\mathrm{t}(48))=2.05$; $\mathrm{p}=0.05)$, depression $(\mathrm{t}(49)=3.91 ; \mathrm{p}=0.001)$ and anxiety $(\mathrm{t}(49)=3.49 ; \mathrm{p}=0.001)$ all remained statistically significant.

Table 1 near here

\section{$\underline{\text { Sessional assessments }}$}

Items on the SRS (relationship with guide, agreement on goals, appropriateness of guides methods and approach, overall session) were consistently high throughout the intervention. Items on the ORS were rated substantially lower at baseline (personal wellbeing Mean=47.2, $\mathrm{SD}=26.1$, interpersonal relationships Mean=59.6, $\mathrm{SD}=23.9$, social relationships Mean=57.2, $\mathrm{SD}=26.7$, and overall wellbeing Mean=50.82, $\mathrm{SD}=21.9$ ) but improved over the course of treatment. 
There were statistically significant correlations between items on the SRS at Session 2 (earlyintervention) and change in Global EDE-Q (Table 2). The correlations were not significant at this time point for ORS items. At Session 4 (mid-treatment) there were significant correlations between all items on the ORS and SRS and change in Global EDE-Q, except for personal and interpersonal wellbeing. At session 6 (late-treatment), agreement on goals was the only sessional rating which was related to outcome.

Table 2 near here

\section{Discussion}

The aim of the study was to determine the preliminary effectiveness of GSH for patients with binge eating delivered by dietitians within a weight management service. Secondly, to determine whether there was an association between sessional ratings of wellbeing, therapeutic relationship and clinical outcomes.

The results of this service evaluation demonstrate that the GSH intervention reduced global eating psychopathology including restraint, eating, shape and weight concern, and reduced depression and anxiety. The scores for shape concern and weight concern at the end of the intervention remained higher than norms of young women in the community [24]. This should be expected given that they were patients with obesity. The improvements in depression are noteworthy in a weight management sample of participants given the bidirectional association between depression and obesity $[25,26]$.

The mean number of objective binge eating episodes reduced by $40 \%$ over the intervention but this did not reach statistical significance. However, an important component of binge eating, the perception of loss of control over eating, did improve. There were no changes in the use of compensatory behaviours such as vomiting, laxative abuse and excessive exercise. Diagnostically, BED excludes these compensatory behaviours. As they were reported at low frequency in this sample we would not expect detectable change.

Improvements in eating disorder psychopathology in the absence of clear reductions in binge eating behaviour may be attributable to several features of the study. First, the duration of the intervention which was brief (6 sessions over 12 weeks), and shorter than many of the 
interventions discussed in the systematic review by Traviss-Turner and colleagues [12]. This review concluded that greater contact time might be necessary for reducing eating disordered behaviours. This is congruent with the experience of the study dietitians who observed a continuation of improvement in the sessions following on from the end of the GSH intervention (those directed primarily at weight management). Second, it should be recognised that this sample of patients were referred for weight management advice. The fact that they were first offered treatment for binge eating may have left some unprepared for the psychological focus on the link between behaviours, thoughts and emotions that the GSH intervention required. Third, this was a relatively small sample of patients which likely lacked power to detect change in a key feature of the disorder.

No significant change in weight was found over the intervention. This is consistent with other interventions aimed at binge eating in patients with obesity which have also reported little or no weight loss [8]. Research by Grilo, et al. [27] has looked at the use of CBT vs behavioural weight loss (BWL) for BED and explored sequencing of treatment. Patients screened for BED and obesity were randomised to one of three groups: CBT for BED (16 x $60 \mathrm{~min}$ sessions over 24 weeks), behavioural weight loss (16 x 60 min sessions over 24 weeks), or CBT then BWL (all sessions over 40 weeks). The CBT and BWL interventions each reduced binge eating. BWL led to greater (but modest and short-term) weight loss. There was no better treatment effect in the group receiving sequential presentation of CBT then BWL. However, remission of binge eating led to greater weight loss and occurred regardless of the type of treatment. This reinforces the idea that binge eating is a barrier to weight loss for at least a proportion of people with obesity, and that further work to address binge eating in weight management settings is merited.

Around half of patients completed the full 12-week intervention. This is in line with the wider GSH literature for eating disorders. Non-completion rates are reported to vary between 1 and $88 \%$, with a median of $59 \%$ for BED [11]. Although rates vary, studies lack robust definitions and reporting of non-completion. Completion rates for community-based weight management programmes typically range from $32-95 \%$, and these interventions tend to be longer [28]. Suggested reasons for non-completion in the GSH literature include the patient feeling better and no longer requiring treatment, poorer psychological well-being and the need for a more complex psychological intervention, or disengagement with the GSH approach due to a conflicting desire to change. The majority of people who withdrew in the current study did so after session one, which suggests that they may not have deemed the 
intervention suitable for them. Furthermore, non-completers scored higher on reported anxiety, eating concern and binge frequency at baseline. It is also difficult to know whether feelings of alienation occurred with the possible unexpected use of eating disorder terminology or being offered a psychological intervention and not a purely dietetic approach as perhaps expected. There is a need to gain a better understanding of when and why patients withdraw from interventions such as this. Additional measures of life events, motivation to change, psychological flexibility as well as dietetic treatment adherence may be useful.

Sessional ratings indicated that the perceived relationship between patients and Guides was consistently high and ratings of well-being improved over the course of the intervention. This contributes to the evidence regarding the acceptability of the intervention being delivered by dietitians. Furthermore, there were associations between sessional ratings mid-treatment and end of treatment outcomes. Improvements in sessional ratings of wellbeing were related to the improvements seen in some measures of eating disorder psychopathology and mood disorder. Previous research has indicated that feedback has a promising effect on maximizing the benefits of outcomes in psychological interventions [29]. However, the benefits of session by session feedback are still inconclusive for patients, as research studies tend to lack homogeneity in outcome, feedback measures and methodology [30].

There are several strengths to this study. This study shows the potential of a GSH intervention for disordered eating in a dietetic-led community weight management service. While reviews suggest that guidance from eating disorders specialists or CBT therapists tend to yield better results than non-specialists, dietitians working in weight management services often undertake additional training in behaviour change and communication skills. Combined with their knowledge of food, physiology and adaptations to diet, this places them in a strong position to facilitate GSH. The dietitians in this study received two days training in being a Guide in GSH that included working with barriers to change. They also had periodic supervision by the authors of the intervention. A further strength is the transdiagnostic approach used the current GSH intervention. Advocates of this approach suggest treating eating disorders in a transdiagnostic manner allows, "for a much more flexible use of therapy, which enables professionals and patients to draw up appropriate formulations and interventions based on the individual's needs rather than being distracted by a formal diagnosis" [31]. Study limitations include this being a single group study with a relatively small number of participants. This limits the generalizability of the findings. In addition, 
there was no formal diagnosis made of BED, only an inclusion assessment of binge eating behavior.

Implications

Reaching a healthy weight is part of the longer-term recovery plan for people with obesity. Having experienced reduced weight, shape and eating concerns, a dietetic department is then in a good position to offer a cohesive pathway of care. At a time when healthcare provision is under pressure these findings are important. With two days additional training and periodic supervision, a brief, evidence-based psychological intervention delivered by dietitians appears to be acceptable and potentially effective for a sub-set of patients with obesity who have symptoms of binge eating. Further research is warranted to understand for whom the intervention is most suitable, to consider longevity in the improvements observed, the impact of these improvements on subsequent weight management, and to formally evaluate costeffectiveness.

\section{Acknowledgments:}

We would like to acknowledge Jonathan Hayes and Vanessa Hunt, the community dietitians who delivered the intervention in Leeds Community Healthcare NHS Trust.

\section{Author contributions:}

Gemma Traviss-Turner - Writing and intellectual input into manuscript. Conducted statistical analyses.

Ursula Philpot - Intervention design, writing and intellectual input into manuscript, training and supervision of dietitians acting as guides.

Janine Wilson - Contribution to design of study, delivery of intervention and data collection. Kathleen Green - Oversaw day to day running of study and analyses.

Suzanne Heywood-Everett - Intervention design, training and supervision of guides and intellectual input into manuscript.

Andrew Hill - Intervention design, writing and intellectual input into manuscript.

\section{Conflict of interest:}


Authors of the manuscript (SHE, AH, UP) were involved in development of the guided selfhelp intervention. Assessments and outcome measures were administered by dietitians who were not involved in this process.

\section{Financial support:}

This research did not receive any external funding.

\section{References:}

1. $\quad$ APA, Feeding and Eating Disorders. 2013: Arlington.

2. Stice, E., C.F. Telch, and S.L. Rizvi, Development and validation of the eating disorder diagnostic scale: A brief self-report measure of anorexia, bulimia, and binge-eating disorder. Psychological Assessment, 2000. 12(2): p. 123-131.

3. Brody, M.J., B.T. Walsh, and M. Devlin, Binge eating disorder: Reliability and validity of a new diagnostic category. Journal of Consulting and Clinical Psychology, 1994. 62: p. 381386.

4. $\quad$ Dailey, S., Gill, C.S, Karl, S.L, et al., DSM-5 Learning Companion for Counsellors. 2014, Alexandria: American Counselling Association.

5. Chao, A.M., Wadden., T.A., Gorin, A.A, et al., Binge eating and weight loss outcomes in individuals with type 2 diabetes: 4-year results from Look AHEAD study. Obesity, 2017. 25: p. 1830-1837.

6. Masheb, R.M., Lutes, L.D., Kim, H.M, et al., High-frequency binge eating predicts weight gain among veterans receiving behavioral weight loss treatments. Obesity, 2015; 23: p. 5461.

7. Network, S.I.G., Management of Obesity: A national clinical guideline. 2010: Edinburgh. p. 19.

8. Berkman, N.D., Brownley, K.A., Peat, C.M., et al. Management and outcomes of bingeeating disorder. Rockville (MD): Agency for Healthcare Research and Quality (US); 2015. Report No.: 15(16)-EHC030-EF.

9. Excellence, N.I.o.H.a.C., Eating Disorders: recognition and treatment. 2017, NICE: London.

10. BEAT. Binge Eating Disorder 2016 [cited 2016 15th March ]; Available from: https://www.b-eat.co.uk/about-eating-disorders/types-of-eating-disorder/binge-eatingdisorder

11. Beintner, I., C. Jacobi, and U.H. Schmidt, Participation and outcome in manualized self-help for bulimia nervosa and binge eating disorder - A systematic review and metaregression analysis. Clinical Psychology Review, 2014. 34(2): p. 158-176.

12. Traviss-Turner, G.D., R.M. West, and A.J. Hill, Guided self-help for eating disorders: A systematic review and metaregression. European Eating Disorders Review, 2017. 25(3): p. 148-164.

13. Traviss, G.D., S. Heywood-Everett, and A.J. Hill, Understanding the 'guide' in guided selfhelp for disordered eating: a qualitative process study. Psychology and psychotherapy, 2013. 86(1): p. 86-104.

14. The British Dietetic Association (BDA), Model for Dietetic Outcomes. 2011: Birmingham. https://www.bda.uk.com/publications/archive/bda_outcome_model_2011_archive

15. Traviss, G.D., S. Heywood-Everett, and A.J. Hill, Guided self-help for disordered eating: A randomised control trial. Behaviour Research and Therapy, 2011. 49(1): p. 25-31.

16. Des Jarlais, D.C., C. Lyles, and N. Crepaz, Improving the Reporting Quality of Nonrandomized Evaluations of Behavioral and Public Health Interventions: The TREND Statement. American Journal of Public Health, 2004. 94(3): p. 361-366.

17. Fairburn, C.G. and S.J. Beglin, Eating disorder examination questionnaire, , in Cognitive behavior therapy and eating disorders, C.G. Fairburn, Editor. 2008, Guilford Press: New York. 
18. Luce, K.H. and J.H. Crowther, The reliability of the Eating Disorder Examination - SelfReport Questionnaire Version (EDE-Q). International Journal of Eating Disorders, 1999. 25(3): p. 349-351.

19. Peterson, C.B., Crosby, R.D., Wonderlich, S.A., et al., Psychometric properties of the eating disorder examination-questionnaire: Factor structure and internal consistency. International Journal of Eating Disorders, 2007. 40(4): p. 386-389.

20. Kroenke, K., R.L. Spitzer, and J.B.W. Williams, The PHQ-15: Validity of a new measure for evaluating the severity of somatic symptoms. Psychosomatic Medicine, 2002. 64(2): p. 258266.

21. Spitzer, R.L., Kroenke, K., Williams, J.B, et al., A brief measure for assessing generalized anxiety disorder: The GAD-7. Archives of Internal Medicine, 2006. 166(10): p. 1092-1097.

22. Miller, S.D. and B.L. Duncan, The Outcome and Session Rating Scales: Administration and scoring manuals. 2000.

23. Campbell, A. and S. Hemsley, Outcome Rating Scale and Session Rating Scale in psychological practice: Clinical utility of ultra-brief measures. Clinical Psychologist, 2009. 13(1): p. 1-9.

24. Fairburn, C.G. and S.J. Beglin, Assessment of eating disorders: Interview or self-report questionnaire? International Journal of Eating Disorders, 1994. 16(4): p. 363-370.

25. Luppino, F.S., De Wit, L.M., Bouvy, P.F, et al., Overweight, obesity, and depression: A systematic review and meta-analysis of longitudinal studies. Archives of General Psychiatry, 2010. 67(3): p. 220-229.

26. Mannan, M., Mamun, A., Doi, S, et al., Is there a bi-directional relationship between depression and obesity among adult men and women? Systematic review and bias-adjusted meta analysis. Asian Journal of Psychiatry, 2016. 21: p. 51-66.

27. Grilo, C.M., Masheb, R.M., Wilson, G.T, et al., Cognitive-behavioral therapy, behavioral weight loss, and sequential treatment for obese patients with binge-eating disorder: A randomized controlled trial. Journal of Consulting and Clinical Psychology, 2011. 79(5): p. 675-685.

28. Hartmann-Boyce, J., Johns, D.J., Jebb, S.A, et al., Behavioural weight management programmes for adults assessed by trials conducted in everyday contexts: Systematic review and meta-analysis. Obesity Reviews, 2014. 15(11): p. 920-932.

29. Schmidt, U., Landau, S., Pombo-Carril, M.G, et al., Does personalized feedback improve the outcome of cognitive-behavioural guided self-care in bulimia nervosa? A preliminary randomized controlled trial. British Journal of Clinical Psychology, 2006. 45(1): p. 111-121.

30. Davidsen, A.H., Landau, S., Pombo-Carril, M.G, et al., Feedback versus no feedback in improving patient outcome in group psychotherapy for eating disorders (F-EAT): Protocol for a randomized clinical trial. Trials, 2014. 15 (1) (138).

31. Waller, G., Cordery, H., Corstorphine, E, et al., Cognitive behavioral therapy for eating disorders: A comprehensive treatment guide. 2007, New York, NY: Cambridge University Press; US. 
Tables

Table 1 Mean (SD) scores on the main outcomes at baseline and end of treatment.

\begin{tabular}{|l|c|c|c|c|c|c|}
\hline \multicolumn{1}{|c|}{ Outcome } & $\begin{array}{c}\text { Baseline } \\
\text { Mean (SD) }\end{array}$ & $\begin{array}{c}\text { End treatment } \\
\text { Mean (SD) }\end{array}$ & $\begin{array}{c}\text { 95\% CI for Mean } \\
\text { Diff. }\end{array}$ & $\boldsymbol{t}$ & df & Sig. \\
\hline $\begin{array}{l}\text { Eating disorder } \\
\text { EDE-Q) }\end{array}$ & & & & & & \\
\hline Restraint & $2.43(1.61)$ & $1.20(1.21)$ & $0.47,1.98$ & 3.34 & 23 & 0.003 \\
\hline $\begin{array}{l}\text { Eating } \\
\text { Concern }\end{array}$ & $2.75(1.46)$ & $1.28(1.43)$ & $0.84,2.11$ & 4.79 & 23 & 0.001 \\
\hline $\begin{array}{l}\text { Shape Concern } \\
\text { Weight Concern }\end{array}$ & $4.54(0.91)$ & $2.55(1.54)$ & $1.34,2.64$ & 6.29 & 23 & 0.001 \\
\hline Global Score & $3.50(1.00)$ & $1.89(1.20)$ & $1.10,2.12$ & 6.48 & 23 & 0.001 \\
\hline $\begin{array}{l}\text { Binge } \\
\text { Episodes }\end{array}$ & $7.80(9.55)$ & $4.76(6.61)$ & $-1.02,7.11$ & 1.55 & 22 & 0.14 \\
\hline Loss of Control & $6.43(8.59)$ & $2.46(3.65)$ & $0.10,7.85$ & 2.13 & 22 & 0.05 \\
\hline $\begin{array}{l}\text { Vomiting } \\
\text { Laxative }\end{array}$ & $0.13(0.46)$ & $0.04(0.21)$ & $-0.09,0.27$ & 1.00 & 22 & 0.33 \\
\hline $\begin{array}{l}\text { Ebuse } \\
\text { Excessive }\end{array}$ & $0.44(1.47)$ & $0.00(0.00)$ & $-0.20,1.07$ & 1.42 & 22 & 0.17 \\
\hline & $2.57(7.05)$ & $0.22(0.85)$ & $-0.76,5.45$ & 1.57 & 22 & 0.13 \\
\hline $\begin{array}{l}\text { Depression } \\
\text { PHQ-9) }\end{array}$ & $13.40(5.56)$ & $6.83(5.71)$ & $3.70,9.42$ & 4.74 & 23 & 0.001 \\
\hline Anxiety (GAD-7) & $10.06(6.04)$ & $5.29(3.64)$ & $2.21,6.87$ & 4.03 & 23 & 0.001 \\
\hline & $43.50(5.04)$ & $42.29(6.74)$ & $-1.32,3.73$ & 1.00 & 19 & 0.33 \\
\hline & & & & & 23 & 0.001 \\
\hline
\end{tabular}


Table 2. Results of correlation analyses in completers, exploring relationship between sessional ratings and outcome.

\begin{tabular}{|c|c|c|}
\hline Sessional item $(\mathrm{N}=\mathbf{2 4})$ & Pearson $r$ & Significance \\
\hline \multicolumn{3}{|l|}{ Session 2 (early) ORS } \\
\hline Personal wellbeing & -.35 & .06 \\
\hline Interpersonal wellbeing & -.21 & .18 \\
\hline Social wellbeing & -.10 & .34 \\
\hline Overall wellbeing & -.28 & .11 \\
\hline \multicolumn{3}{|l|}{ Session 2 (early) SRS } \\
\hline Relationship with guide & -.40 & $.04^{*}$ \\
\hline Agreement on goals & -.45 & $.02 *$ \\
\hline Topic/method & -.41 & $.04 *$ \\
\hline Overall session & -.46 & $.02 *$ \\
\hline \multicolumn{3}{|l|}{ Session 4 (mid) ORS } \\
\hline Personal wellbeing & -.36 & .06 \\
\hline Interpersonal wellbeing & -.31 & .09 \\
\hline Social wellbeing & -.44 & $.02 *$ \\
\hline Overall wellbeing & -.40 & $.04 *$ \\
\hline \multicolumn{3}{|l|}{\begin{tabular}{|l|} 
Session 4 (mid) SRS \\
\end{tabular}} \\
\hline Relationship with guide & -.44 & $.02 *$ \\
\hline Agreement on goals & -.38 & $.05^{*}$ \\
\hline Topic/method & -.48 & $.01 *$ \\
\hline Overall session & -.48 & $.02 *$ \\
\hline \multicolumn{3}{|l|}{ Session 6 (late) ORS } \\
\hline Personal wellbeing & -.24 & .15 \\
\hline Interpersonal wellbeing & -.14 & .28 \\
\hline Social wellbeing & -.18 & .22 \\
\hline Overall wellbeing & -.20 & .19 \\
\hline \multicolumn{3}{|l|}{ Session 6 (late) SRS } \\
\hline Relationship with guide & -.20 & .19 \\
\hline Agreement on goals & -.41 & $.03 *$ \\
\hline Topic/method & -.30 & .10 \\
\hline Overall session & -.17 & .24 \\
\hline
\end{tabular}

* Significant at $\leq 0.05$ 


\begin{tabular}{|c|}
\hline \multicolumn{1}{|c|}{$\begin{array}{c}\text { Consented } \\
\mathrm{N}=50\end{array}$} \\
\hline \multicolumn{1}{|c|}{ Completed Introduction and } \\
Session $1 \mathrm{~N}=50$ \\
Session $2 \mathrm{~N}=37$ \\
Session $3 \mathrm{~N}=31$ \\
Session $4 \mathrm{~N}=28$ \\
Session $5 \mathrm{~N}=26$ \\
Session $6 \mathrm{~N}=24$ \\
\hline Completed primary \\
outcome measures \\
$\mathrm{N}=24$ \\
\hline
\end{tabular}

Figure 1 Flow diagram of participation 


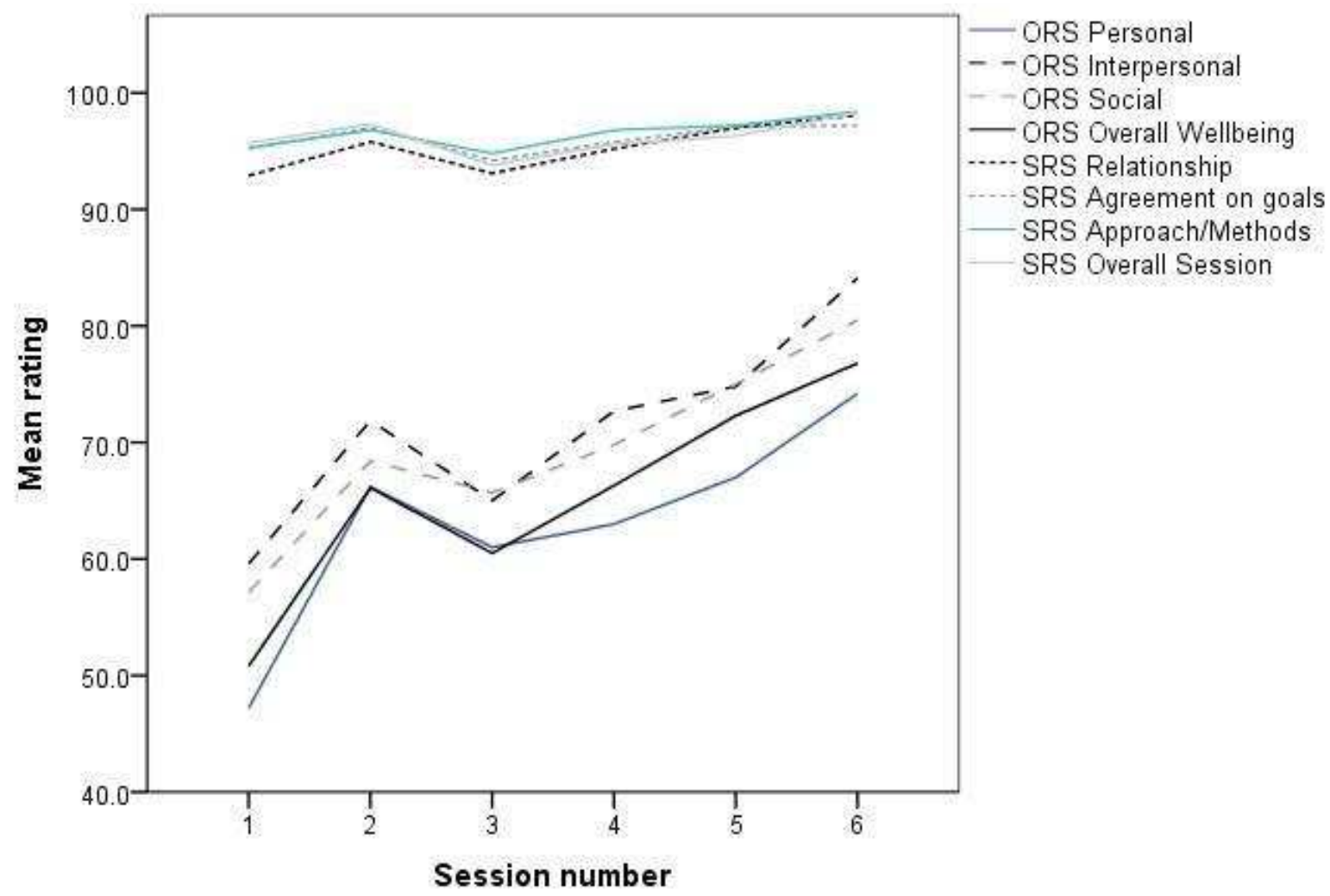

Figure 2. Mean ratings on the Outcome and Sessional Rating Scales by session 